\title{
VIVIENDAS SUPERVISADAS PARA PERSONAS \\ CON TRASTORNO MENTAL SEVERO EN ASTURIAS: \\ ¿AMBIENTE RESTRICTIVO O ABIERTAS A LA COMUNIDAD? 1
}

\section{SUPPORTIVE HOUSING FOR PEOPLE}

WITH SEVERE MENTAL ILLNESS IN ASTURIAS:

RESTRICTIVE ENVIRONMENT OR OPEN TO THE COMMUNITY?

\section{MORADAS SUPERVISIONADAS PARA PESSOAS COM DOENÇA MENTAL GRAVE EM ASTÚRIAS: AMBIENTE RESTRITIVO OU ABERTO PARA A COMUNIDADE?}

\section{Omar García-Pérez}

UNIVERSIDAD DE OVIEDO, ESPAÑA

RESUMEN: A partir del proceso de Reforma Psiquiátrica iniciado en España en 1985 se asume el modelo de atención comunitaria y la filosofía de rehabilitación psicosocial como motor clave para la recuperación personal, social y laboral de las personas con trastorno mental severo. En este sentido, el alojamiento es un componente esencial en la medida que existe evidencia acumulada suficiente para afirmar que la provisión de un alojamiento adecuado representa una de las condiciones críticas para garantizar la atención comunitaria, convirtiéndose en uno de los más importantes programas de apoyo social. Método. El objetivo del estudio es evaluar las viviendas supervisadas para personas con trastorno mental severo en el Principado de Asturias en sus aspectos de infraestructura y el funcionamiento interno de las mismas. Se utiliza la escala Índice Ambiental (IA), y la escala de oportunidad de
Habilidades Básicas de la Vida Diaria (BELS), así como la información aportada por los programas residenciales y los informantes clave de los mismos. Resultados. Las viviendas obtienen de media una puntación en el Índice Ambiental de 17,29 sobre un máximo de 55, lo que le confiere una funcionalidad más abierta que los ambientes institucionales, aunque lejos todavía de las viviendas supervisadas de otros programas residenciales. En síntesis, los resultados otorgan un nivel de independencia considerable para el desempeño de las competencias básicas y sociales de los usuarios. Discusión. Se denota cierto peligro de ambiente institucional en su funcionamiento diario, en aspectos como la red social de los usuarios, formada en gran parte por el personal de las viviendas, el tiempo que llevan residiendo en ellas o su escasa participación e integración social. 
PALABRAS CLAVE: Pedagogía social; Enfermedad mental; Vivienda; Comunidad; Integración social; Educación social.

ABSTRACT: Community care model and philosophy of psychosocial rehabilitation as engine key recovery personal, social and employment of people with severe mental disorder is assumed from the psychiatric reform process begun in Spain in 1985. In this sense, the accommodation is an essential component to the extent that there is sufficient evidence to assert that the provision of adequate accommodation represents a critical condition to ensure community care, becoming one of the most important programs of social support. The aims of the study are to evaluate housing supervised for people with severe mental disorder in Principado of Asturias in its aspects of infrastructure and the inner workings of the same. This uses the Environmental Index (Al), and the scale of opportunity to the Basic Everyday Living Schedule (BELS), as well as the information provided by the residential programs and key informants of the same. Housing obtained from half a score in the environmental index of 17,29 on a maximum of 55 , giving it a more open than the institutional, although far environments functionality yet of the supervised homes of other residential programs. In short, outcomes provide a level of considerable independence to carry out basic and social skills of the users, but true danger of institutional environment in its daily operation, in aspects such as the network of users, formed largely by the staff of the housing, time who is residing in them or their low participation and social integration is denoted.

KEYWORDS: Social Pedagogy; Mental Diseases; Housing; Communities; Social integration; Social education.
RESUMO: Introdução. Do processo de reforma psiquiátrica iniciada em Espanha em 1985, assume-se o modelo de atenção da comunidade e filosofia de reabilitação psicossocial como um fator-chave para a recuperação pessoal, social e de emprego das pessoas com doença mental grave. Neste sentido, a vivenda é um componente essencial na medida em que não é suficiente acumulado evidências para afirmar que a provisão de morada adequada é uma das condições essenciais para garantir cuidados na comunidade, tornando-se um dos mais importantes programas de apoio social . Métodos. O objetivo do estudo é avaliar a habitação supervisionado para pessoas com doença mental grave no Principado das Astúrias, e os aspectos de infra-estrutura do funcionamento interno do mesmo. Use o Índice de escala Ambiental (AI), ea escala de Competências oportunidade Básicas de Vida Diária (BELS), bem como as informações fornecidas pelos programas residenciais e informanteschave do mesmo. Resultados. As propriedades obtidas pelas avaliações médias no Índice Ambiental de 17,29 de um 55 possível, dando-lhe mais funcionalidade aberto a ajustes institucionais, embora ainda longe das casas dos outros programas residenciais supervisionadas. Em resumo, os resultados fornecem um nível de independência considerável para executar habilidades básicas e sociais dos usuários. Discussão. Denota-se algum perigo de ambiente institucional em suas operações diárias, em áreas como a usuários de redes sociais, formados em grande parte por funcionários da Habitação, quanto tempo eles tenham residido eles ou à sua fraca participação e integração social.

PALAVRAS-CHAVE: Pedagogia social; Doença mental; Vivenda; Comunidade; Integração social; Educação social. 


\section{Introducción}

Históricamente, el concepto y atención al trastorno mental ha experimentado diversos giros significativos que han exigido y exigen en la actualidad cambios organizativos de gran calado. En este sentido, la consideración social de las personas con esta problemática ha evolucionado a partir de la transición entre los términos "loco" (que requiere aislamiento y reclusión por su supuesta peligrosidad), "enfermo" (susceptible de atención sanitaria) y "ciudadano" (López Álvarez y Laviana-Cuetos, 2007), que enfatiza su consideración como personas con derechos y deberes, para cuyo ejercicio encuentran dificultades múltiples, derivadas tanto de su enfermedad como de sus consecuencias en términos de discapacidad personal, pero también de la existencia de barreras sociales, ligadas al estigma. En este sentido, esa nueva concepción viene marcada en España por la Reforma Psiquiátrica de 1985, que inicia un proceso de desinstitucionalización y asume el modelo comunitario como marco general de atención, basado en nuevos enfoques rehabilitadores que apuestan por el paradigma de la recuperación (recovery) y bienestar (well-being) (Grupo de trabajo de la Guía de Práctica Clínica de Intervenciones Psicosociales en el Trastorno Mental Grave, 2009). De este modo, se han ido introduciendo conceptos como "atención integral e integrada", que pretenden abarcar todo el déficit y las desventajas sociales que produce el impacto de la enfermedad mental en la persona que la padece (Gómez-Beneyto, 2007, 2011). Así pues, la comunidad adquiere un valor fundamental en el marco de intervención. Esto supone un cambio hacia la participación de diferentes agentes, principalmente socioeducativos y cuyo referente clave es la emancipación y transformación social, y es ahí donde la educación está estrechamente vinculada al desarrollo comunitario (Varela Crespo, 2010).

\section{Modelo de atención comunitaria}

Con el cierre de las grandes instituciones, la vida de las personas con trastorno mental severo se desenvuelve en sus hogares familiares o en residencias y, con ello, sus necesidades se han ido acercando progresivamente a la normalidad, esto es, ajustándose al conjunto de necesidades de la población sana: necesidad de recibir una educación, de trabajar, de poder ganarse la vida, de relacionarse, de ocupar el tiempo libre y, por supuesto, de recibir tratamiento adecuado (Gómez-Beneyto, 2007, 2011). En definitiva, se trata de que estas personas puedan recuperar su proyecto vital. En este punto es donde cobra importancia la rehabilitación psicosocial, pues para conseguir su independencia es necesario contar con una serie de apoyos que faciliten el paso que supone la recuperación. Este proceso se lleva a cabo a través de procedimientos de aprendizaje y provisión de soportes sociales, que sirven de medio para el desarrollo de habilidades personales en los diferentes ámbitos en los que se desenvuelve la persona (Camino Vallhonrat, Hernanz Vaquero y Bosch Vilac, 2010). La atención a las personas con trastorno mental severo requiere la integración de distintos niveles de atención y diferentes tipos de intervención que forman un conjunto inseparable, y que se integran en nuevos objetivos: autonomía, calidad de vida, bienestar personal, participación social en torno al concepto de recuperación personal.

El Principado de Asturias, como refleja el Plan de Salud Mental del Principado de Asturias 2011-2016, coordinado por Amelia González López (2011), se adhiere al modelo de atención comunitaria, siguiendo las directrices europeas pactadas en el Libro Verde de la Unión Europea sobre Salud Mental (2006), la Convención sobre los Derechos de las Personas con Discapacidad de Nueva York, de 13 de diciembre de 2006, así como el modelo nacional desarrollado en la Estrategia en Salud Mental del Sistema Nacional de Salud (2007) y la recién publicada Estrategia en Salud Mental del Sistema Nacional de Salud 2011-2016 (GómezBeneyto, 2011). Por tanto, se ha optado por un marco de intervención que reconoce a la persona con enfermedad mental todos sus derechos y responsabilidades de ciudadano, con actuaciones dirigidas a la normalización e integración plena en la sociedad, evitando su exclusión. 


\section{Atención residencial para personas con trastorno mental severo}

Bajo esta filosofía general de actuación, comienzan a desarrollarse diferentes dispositivos y programas sociosanitarios que faciliten la integración social de las personas con trastorno mental severo en la sociedad. Uno de estos programas es el referido a la atención residencial, en la que están presentes diferentes modelos y estructuras de alojamiento. De este modo, la atención residencial es uno de los últimos eslabones en la normalización deseada por la persona con un trastorno mental, al igual que los programas de inserción laboral, dentro de los programas de apoyo social, que deben estar coordinados con la parte sanitaria del modelo comunitario para una efectiva integración. Por ello, el trabajo de rehabilitación se tornaría sombrío sin el pilar del alojamiento que asegure un mínimo de seguridad y confianza (Gómez, 2002). Sin vivienda estable y de calidad, los equipos comunitarios no pueden funcionar. Tal es su importancia, que Shepherd y Murray sostienen que "la vivienda debe estar en el centro de la psiquiatría comunitaria" (Macpherson, Shepherd, y Edwards, 2004, p.180).

\subsection{Efectos de los alojamientos con apoyo para personas con trastorno mental severo}

Diversos estudios muestran efectos positivos de las viviendas supervisadas y de los programas residenciales en la situación clínica de los usuarios (Fakhoury, Murray, Shepherd y Priebe, 2002; López Álvarez et al., 2005c); en su funcionamiento personal y social (Fakhoury et al. 2002; Hansson et al., 2002; Sörgaard et al., 2001); en su calidad de vida (Hansson et al., 2002; López Álvarez et al., 2005c; Mares, Young, Mcguire y Rosenheck, 2002; Piat y Sabetti, 2011); así como una disminución en el uso de recursos sanitarios (Lascorz, Serrats, Pérez, Fábregas y Vegué, 2009).

Sin embargo, Ogilvie (1997) concluye que vivir en un mal ambiente residencial aumenta el número de servicios necesarios y disminuye la calidad de vida, por lo que se reduce la satisfacción del usuario y se crean mayores necesidades. Asimismo, vivir en un alojamiento adecuado mejora el funcionamiento social, y si se pasa de un alojamiento apropiado a otro inapropiado se produce un deterioro, de modo que estos aspectos indican una clara correlación entre la calidad del alojamiento y la calidad del funcionamiento social. Por otra parte, la inclusión de personas en un alojamiento no adecuado puede reflejar una serie de efectos negativos como el riesgo de institucionalismo, con la aparición y mantenimiento de conductas personales estereotipadas y empobrecidas y escasos contactos sociales (Ogilvie, 1997). A este respecto hay que señalar que, si bien modelos tradicionales y custodiales de funcionamiento se asocian, en general, con peores resultados (Sörgaard et al., 2001), los usuarios no son homogéneos en cuanto a necesidades. Por este motivo, existen grupos de usuarios que se benefician de tipos de funcionamiento diferentes, desenvolviéndose mejor en entornos con mucho personal y bajas exigencias de actividad e interacción, mientras que para otros residentes, un "exceso" de personal provoca la disminución de resultados positivos en términos de autonomía, participación y desarrollo de redes sociales (Fakhoury et al., 2002).

\section{Atención residencial en Asturias}

La evolución de los dispositivos residenciales en Asturias ha tenido una trayectoria discontinua. Por un lado, los años 80 supuso un momento de creación y puesta en marcha de varios dispositivos, dentro del énfasis global del proceso de reforma. Así, en 1987 existían dos pisos protegidos en Avilés; un hogar protegido, denominado "La Casita", para cinco plazas; un programa de ayuda en alojamientos heterofamiliares; "pensiones con veinticinco plazas en Oviedo y un programa de ayuda en viviendas para diez personas en Gijón" (García González, 1988, p.732). En 1993, se contabilizaban 30 plazas entre los pisos protegidos y el hogar protegido "La Casita", pasando a existir 6 pisos protegidos con 17 plazas ocupadas y el hogar tutelado, con capacidad para 7 personas en el año 1998 (Servicio de Salud del Principado de Asturias [SESPA], 1998). A partir de los años 90, la atención residencial sufre un estancamiento y un retroceso ya que, a pesar de que el papel de estos disposi-

[ 126 ] OMAR GARCÍA PÉREZ

SIPS - PEDAGOGIA SOCIAL. REVISTA INTERUNIVERSITARIA [1139-1723 (2013) 22, 126-136] TERCERA ÉPOCA 
tivos residenciales fue muy importante, poco a poco fue decayendo el interés por su utilización (García González y De las Heras, 1998), con el cierre de algunos pisos, debido también a problemas de financiación. Actualmente, existen en funcionamiento "diez viviendas supervisadas para un total de treinta y dos plazas" (González-López, 2011, p.28), si bien la muestra del estudio está compuesta por siete alojamientos. La gestión de los alojamientos es concertada, puesto que la Fundación Asturiana de Atención y Protección a Personas con Discapacidades y/o Dependencias (FASAD) realiza la intervención en seis viviendas, la Fundación SILOÉ en tres pisos y un alojamiento está gestionado directamente por los servicios de Salud Mental.

Así pues, un aspecto vital a la hora de evaluar este tipo de alojamientos es conocer su funcionamiento cotidiano, que nos ofrece información relevante de cómo se entienden las viviendas supervisadas para personas con trastorno mental severo en el ámbito de la atención comunitaria en la red de salud mental pública en Asturias. También nos permite discernir qué tipo de modelo residencial se pretende conseguir y nos da la posibilidad de analizar las diferencias existentes de concepto y de programa residencial entre las distintas fundaciones o servicios que prestan el apoyo y supervisión de los dispositivos residenciales.

\section{Método}

La investigación que en estas páginas se aborda forma parte de un estudio más amplio que trata de evaluar las viviendas supervisadas existentes para personas con trastorno mental severo en el Principado de Asturias. Sus objetivos son analizar las características generales de los alojamientos y el funcionamiento básico y social de sus usuarios, así como su apoyo social comunitario, su calidad de vida y sus actitudes y grado de satisfacción respecto a la vivienda. Los resultados específicos que aquí se muestran son los derivados del funcionamiento interno de las viviendas, que se asumen como el último escalón en el proceso de la rehabilitación psicosocial de sus usuarios, requiriendo del ámbito pedagógico para su desarrollo, intervención y evaluación.

En este sentido, se trata de una investigación orientada al cambio, a la mejora y a la transformación social, a la que "la evaluación puede contribuir ayudando a las instituciones democráticas a seleccionar, supervisar, mejorar y dar sentido a los programas y a las políticas sociales" (Mark, Henry y Julnes, 2000, p.3). Asimismo, la investigación es participativa, fundamentalmente descriptiva y de cédula única. El método seleccionado para el trabajo de campo es "cuanticualitativo" (Barrón, Bas Peña, Crabay y Schiavoni, 2010, p.86), a través de diversas encuestas, análisis documental y notas de campo realizadas durante la investigación. La técnica básica de recogida de información es la entrevista, principal recurso metodológico a utilizar en el proceso de evaluación. Esto es así porque la rehabilitación psicosocial tiene como característica principal su individualidad, derivada de la particularidad de las personas a evaluar y de los contextos donde éstas interactúan o se prevé que vayan a interactuar. Estas circunstancias colocan a la entrevista como el método más adecuado de recabar información en rehabilitación (Fernández, González Cases, Mayoral y Touriño, 2003).

La elección de la muestra ha sido a través de un muestreo no probabilístico, a conveniencia, de modo que la muestra englobaba a toda la población objeto de estudio existente en el Principado de Asturias: las siete viviendas supervisadas para personas con trastorno mental severo y sus usuarios. Cuatro de ellas gestionadas por FASAD, dos por SILOÉ y una por los servicios de Salud Mental de la zona en la que se encuentra la vivienda.

Para su análisis concreto se utilizaron, principalmente, dos instrumentos de recogida de información:

a) Indice Ambiental (El-Environmental Index). Esta escala está adaptada, con varias preguntas adicionales, del Hospital and Hotel Practices Profile de Wykes 1982 (Jordá y Espinosa, 1990, 1991). Su objetivo es valorar el grado de autonomía de un individuo dentro de su entorno residencial y evaluar la calidad y las actividades disponibles en la localidad. La escala está dividida en subsecciones con los encabezamientos de: Actividad, Pertenencias, Comidas, Salud e Higiene, Habitaciones de los Residentes, Servicios y Entorno. Está diseñada para uso tanto en hospitales, como para todo tipo de alojamientos con personal donde sus usuarios sean residentes con trastorno mental. Consta de 55 ítems cuya categoría de respuesta es sí o no. La puntuación obte- 
nida, con un máximo de 55, más que el nivel de "restrictividad" o "restricción", como se denomina en la versión original del instrumento (Jordá y Espinosa, 1990; Maestro Barón et al., 2001; O 'Driscoll y Leff, 1993; Rickard et al., 2002), parece medir, de manera más general, la mayor o menor proximidad funcional a contextos o ambientes institucionales (López Álvarez et al., 2005b). Asimismo, ha demostrado ser un cuestionario adecuado para medir la adecuación de ajuste entre las necesidades del paciente y las instalaciones residenciales. Esto debería ayudar a dirigir a las personas con trastornos mentales graves y persistentes hacia las instalaciones mejor adaptadas a sus necesidades (Corbiere, Lesage, Reinharz y Contandriopoulos, 2001). Esta entrevista ha sido realizada a las personas responsables de las viviendas para personas con TMS.

b) Habilidades Básicas de la Vida Diaria (BELS-Basic Everyday Living Schedule) Originalmente elaborado por Julian Leff, el BELS es un instrumento diseñado para evaluar las habilidades específicas básicas de convivencia diaria en personas que sufren un trastorno mental de larga duración (Leff, Trieman y Gooch, 1996). Se administra a un informante clave, en este caso el personal de las viviendas y explora cuatro áreas fundamentales de funcionamiento: 1) autocuidado, 2) habilidades domésticas, 3) habilidades comunitarias, y 4) actividad y relaciones sociales. Permite puntuar el desempeño o ejecución actual del sujeto y la oportunidad que tiene para actuar de forma independiente en cada área. El cuestionario se compone de 26 ítems. Cada uno de ellos se divide en dos escalas, una de oportunidad de ejecución y otra de desempeño de la habilidad. En este caso, se utilizará la escasa de oportunidad, de tres niveles para determinar el grado de oportunidad que la persona tiene para la realización de actividades de forma independiente: total oportunidad de independencia (2 puntos), alguna oportunidad de independencia (1 punto) y, ninguna oportunidad de independencia (o puntos). En España existe un estudio de fiabilidad realizado con una muestra de 77 pacientes mentales crónicos alojados en pisos y casas-hogar protegidas, ubicadas en Granada y Sevilla (Jiménez et al., 2000). Los resultados del análisis de fiabilidad del estudio muestran, en general, una concordancia global muy buena tanto en la escala de Oportunidad (valor medio de 0.791) como en la de Ejecución ( valor medio de 0.743).

La administración de las entrevistas se realizó de forma individual en las propias viviendas supervisadas objeto de estudio. Para ello, se obtuvieron los permisos necesarios de todos los agentes implicados en la investigación, tanto por los servicios de Salud Mental, como por las entidades que gestionan las viviendas y sus usuarios tras consentimiento informado. Su aplicación fue llevada a cabo por un único entrevistador entrenado para tal fin, con el objetivo de evitar posibles desviaciones en las interpretaciones. Al mismo tiempo, se realiza un análisis documental de la información recogida de los servicios de Salud Mental y de los programas de atención residencial de las organizaciones gestoras de las viviendas.

\section{Análisis e interpretación de los resultados}

\subsection{Aspectos de Infraestructura}

En primer lugar, mostraremos los aspectos de infraestructura de las viviendas analizados, como el número de plazas de cada dispositivo, la modalidad de dormitorios, un aspecto considerado importante por los usuarios por cuestiones personales de intimidad, así como la ubicación y los servicios del entorno.

De este modo, los pisos en Asturias tienen una media de 3,06 plazas, pudiendo ampliarse plazas en algún piso, al disponer de habitación libre. En cuanto a la modalidad de dormitorios, el $94 \%$ de los pisos están ocupados por los usuarios en habitaciones individuales. Asimismo, los lugares elegidos para el desarrollo de las viviendas marcan una distribución territorial mayoritariamente urbana y un enclave bastante razonable, con un fácil acceso a instalaciones comerciales, cafeterías, centros sociales, cines, etc. (Tabla 1).

Por tanto, estos datos indican que la ubicación general de las viviendas tiene lugar en entornos vecinales normales, con las correspondientes posibilidades de interacción social y vida comunitaria, dándose las condiciones adecuadas de infraestructura para una integración social plena.

[ 128 ] OMAR GARCÍA PÉREZ

SIPS - PEDAGOGIA SOCIAL. REVISTA INTERUNIVERSITARIA [1139-1723 (2013) 22, 126-136] TERCERA ÉPOCA 
Tabla 1. Ubicación y entorno de las viviendas supervisadas

\begin{tabular}{|c|c|c|}
\hline & Vivienda Supervisada & \\
\hline \multirow[t]{2}{*}{ Ubicación } & Urbana & $85,7 \%$ \\
\hline & Rural & $14,3 \%$ \\
\hline \multicolumn{3}{|l|}{ Entorno } \\
\hline \multirow{4}{*}{$\begin{array}{l}\text { Servicios del entorno que se encuentran a } \\
\text { una distancia a la que se puede ir caminando }\end{array}$} & Instalaciones comerciales & $100 \%$ \\
\hline & Bares y cafeterías & $100 \%$ \\
\hline & Centros sociales & $85,7 \%$ \\
\hline & Cines & $85,7 \%$ \\
\hline
\end{tabular}

Fuente: elaboración propia

Tabla 2. Funcionamiento interno de las viviendas

\section{Factores}

Normas restrictivas

\section{Pautas concretas}

Restricción para salir por noche

Hora de levantarse establecida

Hora determinada de vuelta

Prohibición de fumar en dormitorios

Visitas establecidas

Límite horas ver TV

Horas de comida establecidas

Hora de acostarse fijada

Restricción para ir al bar

Horas de baño establecidas

Prohibición tener cerillas, mecheros

Restricción para salir durante día

Puerta de la vivienda cerrada

Prohibición de posesión de cuchillas, cuchillos, tijeras

Limitación para la autonomía

Limitación para tener medicamentos

Limitación para cerrar la puerta del baño con llave

Posibilidad de entrar el personal sin permiso en habitaciones

Limitación para tener mobiliario propio o artículos de adorno

Participación

\begin{tabular}{|c|c|c|}
\hline & Posibilidad de entrar el personal sin permiso en habitaciones & $4(57,1 \%)$ \\
\hline & Limitación para tener mobiliario propio o artículos de adorno & $2(28,6 \%)$ \\
\hline \multirow[t]{6}{*}{ Limitación a la privacidad } & Deber de decir a dónde van cuando salen & $2(28,6 \%)$ \\
\hline & Limitación para tener el dinero personal & $1(14,3 \%)$ \\
\hline & Limitación para hacerse café o té & $\bigcirc(\bigcirc \%)$ \\
\hline & Limitación para hacer comidas ligeras & $\bigcirc(\bigcirc \%)$ \\
\hline & Limitación para tener pertenencias bajo llave & $\bigcirc(\bigcirc \%)$ \\
\hline & Personal supervisa pertenencias & $\bigcirc(\bigcirc \%)$ \\
\hline \multirow[t]{2}{*}{ Participación } & Participan en planificación y preparación de comidas & $7(100 \%)$ \\
\hline & Reuniones semanales personal-residentes & $4(57,1 \%)$ \\
\hline
\end{tabular}

Viviendas supervisadas

\begin{tabular}{l}
$7(100 \%)$ \\
\hline $7(100 \%)$ \\
\hline $7(100 \%)$ \\
\hline $7(100 \%)$ \\
\hline $4(57,1 \%)$ \\
$4(57,1 \%)$ \\
\hline $4(57,1 \%)$ \\
\hline $3(42,9 \%)$ \\
\hline $2(28,6 \%)$ \\
\hline $2(28,6 \%)$ \\
\hline$(14,3 \%)$ \\
\hline$(0 \%)$ \\
\hline$(0 \%)$ \\
\hline$(0 \%)$ \\
\hline
\end{tabular}

$4(57,1 \%)$

İndice Global (Máxima restricción: 55)

Media: 17,29 


\subsection{Funcionamiento interno de las viviendas}

En cuanto al funcionamiento interno de las viviendas, se muestran los resultados del Índice Ambiental englobados en varias dimensiones de interés, tales como las normas restrictivas, la limitación para la autonomía, la limitación a la privacidad (Tabla2).

En general, la tabla refleja una relativa "normalización" del funcionamiento, con un valor medio del índice global de 17,29 (DT=2,49) aunque con variaciones internas en función de la entidad que proporciona la supervisión de los pisos, y un número mejorable de casos en los que se registran normas restrictivas, ya que algunos de los datos resultan llamativos por las diferencias que marcan con respecto a lo que cabe imaginar como "ambiente de hogar", más propio de este tipo de dispositivos residenciales. Así, las mayores restricciones vienen motivadas por aspectos de horarios, salidas al exterior de las viviendas o limitaciones en cuestión a la medicación, tema muy importante para el bienestar funcional de las personas con trastorno mental severo, por lo que todos los pisos optan por tener un control directo en el suministro y toma de la misma.

Por otro lado, las puertas están abiertas en el conjunto de las viviendas, sin restricciones para salir durante el día, y no existen demasiadas limitaciones en aspectos básicos de la vida en cuanto a la autonomía y privacidad de los residentes, si bien coexisten en los diferentes alojamientos algunas limitaciones que parecen indicar unos ambientes más institucionales que hogareños, como el hecho de tener que decir al personal a dónde van cuando salen de casa ( $28,6 \%$ de los pisos). Asimismo, sorprende la circunstancia de que el personal puede entrar en las habitaciones de los usuarios sin permiso en el 57,1\% de las viviendas, aunque este porcentaje, a primera vista, resulta engañoso ya que no se trata de una limitación a la privacidad propiamente dicha del programa, sino que se debe más a la confianza adquirida entre el personal y los residentes y a un ambiente familiar más que a una limitación. Por otra parte, hay reuniones semanales entre el personal y los usuarios en el 57,1\% de los pisos, aunque este aspecto se puede valorar desde dos perspectivas contrarias: las reuniones son formalmente institucionales, pero parecen ser muy útiles para regular muchos aspectos de la vida colectiva y para limar detalles del ambiente interno de las viviendas.

La información del aspecto funcional de los alojamientos se completa con la derivada de la aplicación de la escala de oportunidad del BELS, que describe la posibilidad que cada residente tiene para desarroIlar determinadas habilidades cotidianas en el alojamiento que reside, y cuya valoración es previa a la de su capacidad real para desempeñarla (Tabla 3).

Los datos reflejados en la tabla anterior matizan y completan las pautas estudiadas en el índice ambiental de las viviendas. En este sentido, se observa de modo claro la total libertad con la que cuentan los usuarios de los alojamientos para salir durante el día, siendo las viviendas dispositivos de régimen abierto. De este modo, los usuarios son libres para elegir sus actividades de ocio y tiempo libre, para salir y entrar de la vivienda y realizar todo tipo de contactos y relaciones sociales con las personas de su entorno. Igualmente, tampoco se restringen la mayor parte de las acciones que engloban los factores de autocuidado, ni se limita la oportunidad de ejercer el desempeño de sus habilidades domésticas y comunitarias ni sociales.

A través de esta escala se configura, generalmente, un alojamiento abierto, con restricciones moderadas o significativas en apartados ya expresados en el índice ambiental, como en el ítem despertarse $y$ levantarse, o el de la vuelta a casa por la noche ya que, aunque se trata de un alojamiento de régimen eminentemente abierto, deben informar o pedir permiso para poder salir y establecer un pacto sobre la hora de vuelta.

El resto de variables en las que no tienen total libertad para desempeñarlas son las referentes a la medicación, a la compra de comestibles, al uso de servicios asistenciales y a la capacidad para manejar su propio dinero o presupuesto, aunque estas restricciones, sean significativas o moderadas, dependen más del nivel de desempeño del usuario, de su capacidad para realizar de forma independiente tales acciones, que de la propia normativa impuesta por el piso o programa residencial al que se adhiere.

[ 130 ] OMAR GARCÍA PÉREZ

SIPS - PEDAGOGIA SOCIAL. REVISTA INTERUNIVERSITARIA [1139-1723 (2013) 22, 126-136] TERCERA ÉPOCA 
Tabla 3. Porcentaje de usuarios según el grado de oportunidad para la realización de actividades de forma independiente

\begin{tabular}{|c|c|c|c|}
\hline \multirow[t]{2}{*}{ Áreas o actividades } & \multicolumn{3}{|c|}{ Viviendas supervisadas } \\
\hline & $\begin{array}{l}\text { Restricción } \\
\text { significativa }\end{array}$ & $\begin{array}{l}\text { Restricción } \\
\text { moderada }\end{array}$ & $\begin{array}{l}\text { Ausencia de } \\
\text { restricción }\end{array}$ \\
\hline Independencia de movimientos & 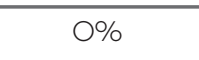 & ০\% & $100 \%$ \\
\hline Despertarse y levantarse & O\% & $28,6 \%$ & $71,4 \%$ \\
\hline Vestirse & О\% & ○\% & $100 \%$ \\
\hline Vuelta a casa por la noche & О\% & $100 \%$ & O\% \\
\hline Comidas & ০\% & ○\% & $100 \%$ \\
\hline Medicación & $23,8 \%$ & $61,9 \%$ & $14,3 \%$ \\
\hline Higiene personal & ○\% & ০\% & $100 \%$ \\
\hline Ropa & O\% & O\% & $100 \%$ \\
\hline Incontinencia & O\% & O\% & $100 \%$ \\
\hline Comportamiento en el cuarto de baño & O\% & O\% & $100 \%$ \\
\hline Preparación de comidas & O\% & $\mathrm{O} \%$ & $100 \%$ \\
\hline Preparación de comidas simples & O\% & O\% & $100 \%$ \\
\hline Compra de comestibles & $28,6 \%$ & $9,5 \%$ & $61,9 \%$ \\
\hline Compras & ০\% & O\% & $100 \%$ \\
\hline Lavado de ropa & O\% & O\% & $100 \%$ \\
\hline Cuidado de espacio personal & O\% & O\% & $100 \%$ \\
\hline Cuidado de su casa o zonas comunes & O\% & O\% & $100 \%$ \\
\hline Uso de transporte público & $\mathrm{O} \%$ & $\mathrm{O} \%$ & $100 \%$ \\
\hline Uso de servicios asistenciales & $19,1 \%$ & $23,8 \%$ & $57,1 \%$ \\
\hline Uso de los locales y servicios públicos & ০\% & ০\% & $100 \%$ \\
\hline Uso de dinero o capacidad de controlar su presupuesto & O\% & $23,8 \%$ & $76,2 \%$ \\
\hline Ocupación diaria & O\% & ○\% & $100 \%$ \\
\hline Actividades de tiempo libre & O\% & O\% & $100 \%$ \\
\hline Sociabilidad & О\% & О\% & $100 \%$ \\
\hline Consideración y preocupación por otros & O\% & O\% & $100 \%$ \\
\hline Prestar auxilio en una emergencia & O\% & O\% & $100 \%$ \\
\hline
\end{tabular}

Fuente: elaboración propia

Otro aspecto en el que existe algún tipo de restricción es en la variable uso de servicios asistenciales, tanto sanitarios como sociales (médico general, dentista, seguridad social, etc.). En este caso, desde las viviendas se espera que concierten sus propias citas con total independencia aunque, en muchas ocasiones, es el personal quien lo efectúa. Sin embargo, en el funcionamiento y desarrollo cotidiano de la convivencia, el personal siempre está atento y suele conocer prácticamente todas las citas de los usuarios, al igual que supervisa o apoya en todas aquellas cuestiones de la vida diaria, aunque exista total independencia por parte de los residentes. 


\section{Conclusiones y discusión}

En primer lugar, en cuanto al número total de dispositivos en Asturias, si comparamos las tasas de plazas con las existentes en otras Comunidades Autónomas, como Andalucía, Madrid o Castilla-La Mancha, por ejemplo, el balance es netamente negativo. Del mismo modo, la dotación de recursos residenciales en Asturias, de 2,04 plazas por 100.000 habitantes es, a toda luz, insuficiente si la comparación se realiza con el ratio de plazas por 100.000 habitantes que la Asociación Española de Neuropsiquiatría (AEN) propone. En su caso, el documento técnico que sirve de consenso, establece un mínimo de 20 y un óptimo de 50 plazas por 100.000 habitantes. Sin embargo, considerando que este es un escenario ideal y, dada la realidad actual, estiman razonable plantear como "apropiado 10 plazas de mínimo y 20 como estimación óptima" (AEN, 2002, p.59). Aunque en el desarrollo de otros programas estatales de referencia como el de la Comunidad de Madrid (Florit Robles, Cañamanes Yelmo, Collantes Olmeda y Rodríguez González, 2007) y Castilla-La Mancha- FISLEM- (Contreras Nieves, Navarro Bayón y Domper Tornil, 2007), marcan un mínimo de 6 plazas por 100.000 habitantes. Sin embargo, el Plan de Salud Mental del Principado de Asturias 2011-2016, estima las necesidades de alojamiento, para diferentes grados de apoyo o supervisión, en valores aproximados a 143 plazas por cada 100 mil habitantes. Sumado a ello, marcan el objetivo, según estimación de necesidades de alojamiento, el tener en funcionamiento 19 viviendas supervisadas o pisos con diferentes intensidades de apoyo que alberguen a 95 usuarios, así como la creación de 3 miniresidencias con 76 plazas totales (González-López, 2011).

Además, si el referente es el de otros países europeos, la situación se agrava considerablemente. Lógicamente, ninguna de las cifras de los estudios y países europeos, como los realizados en Copenhague, Ámsterdam, Londres o Verona (López Álvarez et al., 2004) puede extrapolarse sin más a la presente investigación y no está claro que la evolución de factores de tipo sociocultural vayan a desarrollarse en idéntica dirección, ni mucho menos a un ritmo predecible (López Álvarez et al., 2005b). Sumado a ello, casi ningún usuario se ve obligado a compartir habitación, aunque es señalado como un elemento de descontento por quienes lo sufren; incluso puede llegar a tener consecuencias en la convivencia y en el nivel de apoyo social de los sujetos, puesto que una serie de estudios de Baum y colaboradores (Evans, Wells \& Moch, 2003) demuestran que el diseño de habitación doble tiene efectos negativos sobre el apoyo social de los residentes, en contrapartida con los usuarios alojados en habitaciones individuales.

Por otro lado, la situación de los dispositivos actuales, también en términos de acceso a servicios comunitarios, parece traducir una ubicación de los mismos en entornos residenciales normales, lo que favorece la integración y mejora la calidad de vida de los residentes, tal y como muestran diversos estudios (Contreras Nieves et al., 2007; De Girolamo et al., 2007; Florit Robles et al., 2007; Kyle y Dunn, 2008; López Álvarez et al., 2004, 2005a; Mares et al., 2002; Rickard et al., 2002; Shepherd y Murray, 2001). Sin embargo, la utilización real que hacen de estos servicios es muy escasa, como demuestra la baja puntuación que obtienen los usuarios de las viviendas en participación e integración comunitaria.

La puntuación media en el Índice Ambiental $(17,26)$, marca una cierta normalización respecto al ambiente restrictivo interno de las viviendas si lo comparamos, por ejemplo con la puntuación de las Casas Hogar del programa residencial de la Fundación Pública Andaluza para la Integración Social de Personas con Enfermedad Mental (FAISEM) (Fernández Portes, 2008; López Álvarez et al., 2005b), con una media de 22,7 restricciones o limitaciones; aunque francamente mejorables si la medida comparativa son las viviendas supervisadas de este mismo programa, que alcanza una media de 13,2 limitaciones para la autonomía de sus usuarios. Sin embargo, ambos programas se encuentran lejos del ambiente normalizado que se encuentra en los dispositivos residenciales de este tipo en Londres, con una media valorativa en el índice ambiental de 6,2 medidas restrictivas (Rickard et al., 2002). No obstante, el modelo residencial asturiano, tiene una serie de pautas diferentes motivadas por los distintos programas que llevan a cabo las entidades encargadas en la supervisión y apoyo de las viviendas, siendo parecido, en algunas intervenciones, al desarrollo residencial en Italia, con mayor dependencia de las estructuras sanitarias. Del mismo modo, llama la

[ 132 ] OMAR GARCÍA PÉREZ

SIPS - PEDAGOGIA SOCIAL. REVISTA INTERUNIVERSITARIA [1139-1723 (2013) 22, 126-136] TERCERA ÉPOCA 
atención la diversidad de situaciones dentro de cada tipo de dispositivo y la incongruencia que se da, en ocasiones, entre la puntuación del ambiente restrictivo y su funcionamiento real del día a día. Aún así, en conjunto, da la impresión de que sigue habiendo, en más ocasiones de las debidas, respuestas espontáneamente institucionales que tienden a perpetuarse si no se combaten diariamente. En este sentido, parece haber cierta indefinición en los programas a la hora de objetivar los fines del alojamiento, es decir, si se trata de un recurso de entrenamiento en habilidades y competencias con un nivel de apoyo más intensivo o viviendas con un apoyo más puntual. Este hecho provoca que, en muchas ocasiones, los profesionales de apoyo ejerzan una intervención más restrictiva, cronificando la connotación de enfermedad y dependencia.

Sumado a ello, se considera que la configuración de estructuras institucionalizadas demuestra ser la opción menos favorable, a tenor de los resultados obtenidos en otros estudios acerca de las diferencias de efectividad de distintos tipos de dispositivos residenciales. En este sentido, Kallert, Leisse y Winiecki (2007), sostienen que se debe mantener y fomentar una situación de vida cotidiana en los pacientes y promover el contacto social regularmente. De este modo, para evitar la propagación de los fenómenos de reinstitucionalización (Priebe et al., 2005), las políticas de salud deben promover la inversión en viviendas subvencionadas y abogar por tratar de mejorar el funcionamiento autónomo de los pacientes con esquizofrenia en sus propios alojamientos.

Se aprecia, por otro lado, cierto peligro de institucionalización en los distintos programas residenciales, puesto que, en gran medida, como demuestran otras conclusiones de la investigación general, el círculo de apoyo social queda reducido al personal del alojamiento y a los compañeros de piso, creando un microcosmos que reduce su participación comunitaria. Otro elemento que puede argumentar ese peligro real de institucionalización es el hecho de que el $28,6 \%$ de los usuarios del programa residencial llevan más de dos años viviendo en los pisos con apoyo. Esos dos años se considera la fecha límite establecida, a priori, para su salida del programa y su inserción en alojamientos independientes, puesto que estas viviendas nacieron con la idea de "entrenamiento" y aprendizaje de sus usuarios que les sirviera de anclaje en la comunidad, como un vehículo de enseñanza-aprendizaje de todas las habilidades y competencias necesarias para su real inserción y participación comunitaria. Por tanto, a raíz de la imposibilidad de encontrar vías residenciales alternativas, se cambia la configuración de las viviendas de temporales a permanentes en algunas de las analizadas en el estudio. Por tanto, a pesar del enfoque de rehabilitación de estas instalaciones, y las crecientes expectativas de los cuidadores, las bajas tasas de integración comunitaria o de independencia residencial han sido una tónica habitual en programas también de otros países, como EE.UU., Italia y Gran Bretaña (Piat y Sabetti, 2011). En este sentido, es necesaria la creación de actividades externas, cursos y actividades de formación que partan de sus propios intereses, sirviendo el personal de la vivienda como enlace para este tipo de proyectos. Sin embargo, un estudio de 2009 elaborado por Bitter, Entenfellner, Matsching, Frottier y Frühwald, realiza una revisión sobre diferentes investigaciones que analizan esta cuestión entre 1997 y 2007, llegando a la conclusión de que el peligro de trans-institucionalización sigue vigente, no encontrando evidencia sobre modelos de apoyo adaptados a las necesidades individuales de los pacientes.

Con todo ello, las personas con trastorno mental severo pueden vivir satisfactoriamente en la comunidad con un adecuado alojamiento y un adecuado apoyo (Ogilvie, 1997; Kyle y Dunn, 2008), pero se necesita que las políticas públicas promuevan el éxito, velando porque todos los apoyos necesarios estén disponibles y realicen un seguimiento y evaluación de todo el proceso e intervención, en donde el aspecto educativo se configura clave en la adquisición de competencias y habilidades sociales, muy deficientes en los usuarios de las viviendas en Asturias, y de desarrollo personal, así como en aspectos de orientación e inserción laboral. En este sentido, estamos de acuerdo con lo expresado por Newman y Goldman (2008), en un artículo referido a la implementación de políticas públicas en salud mental, en donde remarcan que la tendencia general en cuanto a atención residencial es abrir los dispositivos y luego ya se pensará lo que se realiza en ellos. Estos autores concluyen que la experiencia ha determinado que las necesidades de alo- 
jamiento de estas personas son, muchas veces, preeminentes, que den sentido a una satisfacción inmediata pero que, sin embargo, se deben buscar soluciones permanentes y programas de intervención eficaces, aceptando la creación de viviendas para atender la gravedad de la situación, pero que el trabajo futuro se desarrolle de una forma persistente, tal y como expresan en 2008 Newman y Goldman en su elocuente escrito titulado "Putting housing first, making housing last".

Así pues, la finalidad última de los servicios residenciales para personas con trastorno mental severo es servir como punto de partida para conseguir el cambio de rol de "cliente" a un papel de ciudadano por medio de la vivienda, así como mediante la educación y las oportunidades de trabajo (Piat y Sabetti, 2011). Esta es la continuidad de la recuperación. Desde esta perspectiva, las viviendas supervisadas no promueven la recuperación, ni la participación o integración social, ni la mejora de sus competencias por sí mismas, por el hecho de ser el recurso residencial con menor nivel de supervisión y más independiente, sino que ha de reflejar la elección de los usuarios, y ha de cumplir con los requisitos específicos de avance de la recuperación para cada individuo. A partir de tal concepción, la comunidad debe ser el referente fundamental de la acción educativa.

\section{Referencias Bibliográficas}

AEN (2002). Rehabilitación psicosocial del trastorno mental severo. Situación actual y recomendaciones. Cuadernos Técnicos, 6. Madrid: AEN.

Barrón, M., Bas Peña, E., Crabay, M.I. y Schiavoni, M.C. (2010). Adolescentes, violencia y familia en la ciudad de Córdoba (Argentina). Pedagogía Social. Revista Interuniversitaria, 17, pp. 83-95.

Bitter, D., Entenfellner, A., Matschnig, T., Frottier, P. y Frühwald, S. (2009). At home in a home? Did de-hospitalization mean de-institutionalization? Psychiatrische Praxis, 36 (6), pp. 261-269.

Camino Vallhonrat, A., Hernanz Vaquero, A. y Bosch Vilac, M. (2010). Piso protegido: ¿̇un medio o un fin en la rehabilitación psicosocial? Revista de la Asociación Española de Neuropsiquiatría, 30 (106), pp. 279-290.

Contreras Nieves, J.A., Navarro Bayón, D. y Domper Tornil, J.A. (2007). Programas de rehabilitación psicosocial y apoyo comunitario para personas con enfermedad mental. Toledo: Fundación FISLEM.

Corbière, M., Lesage, A.D., Reinharz, D. y Contandriopoulos, A. (2001). A French, abridged version of the Hospitals and Hostels Practices Profile Schedule. International Journal of Methods in Psychiatric Research, 10 (4), pp. 183-190.

De Girolamo, G. et al. (2007). The current state of mental health care in Italy: problems, perspectives, and lessons to learn. European Archives of Psychiatry and Clinical Neuroscience, 257, pp. 83-91.

Evans, G., Wells, N. y Moch, A. (2003). Housing and mental health: a review of the evidence and methodological and conceptual critique. Journal of social issues, 59 (3), pp. 475-500.

Fakhoury, W., Murray, A., Shepherd, G. y Priebe, S. (2002). Research in supporting housing. Social Psychiatry and Psychiatric Epidemiology, 37, pp. 301-315.

Fernández, J., González Cases, J., Mayoral, F. y Touriño, R. (2003). Evaluación funcional en rehabilitación psicosocial. Métodos e instrumentos. En C. Gisbert. Rehabilitación psicosocial y tratamiento integral del trastorno mental severo, (pp.57106). Madrid: AEN.

Fernández Portes, L. (2008). Programas residenciales en la atención comunitaria a personas con trastorno mental grave. Estructuras de alojamientos, nuevos perfiles. Una visión desde Andalucía. /l Congreso de la Federación Española de Asociaciones de Rehabilitación Psicosocial. Bilbao 5, 6 y 7 de Junio de 2008. Inédito.

Florit Robles, A., Cañamares Yelmo, J.M., Collantes Olmeda, B. y Rodríguez González, A. (2007). Atención Residencial Comunitaria y Apoyo al Alojamiento de Personas con Enfermedad Mental Grave y Crónica: Recursos Residenciales y Programas Básicos de Intervención. Madrid: Consejería de Familia y Asuntos Sociales.

García González, J. (1988). La cuestión de la desinstitucionalización y de la Reforma Psiquiátrica en Asturias: cinco años de evolución (1983-1987). Revista AEN, VIII, 27, pp. 723-749.

García González, J. y De Las Heras, B. (1998). Soportes para la convivencia y la integración social de pacientes con trastornos persistentes: funciones y necesidades. En J. García González, A. Espino Granado y L. Lara Palma (ed.). La psiquia-

[ 134 ] OMAR GARCÍA PÉREZ

SIPS - PEDAGOGIA SOCIAL. REVISTA INTERUNIVERSITARIA [1139-1723 (2013) 22, 126-136] TERCERA ÉPOCA 
tría en la España de fin de siglo. Un estudio sobre la reforma psiquiátrica y las nuevas formas de atención en salud mental, (pp. 225-239). Madrid: Díaz de Santos.

Gómez-Beneyto, M. (coord.) (2007). Estrategia en Salud Mental del Sistema Nacional de Salud, 2006. Madrid: Ministerio de Sanidad y Consumo.

Gómez-Beneyto, M. (coord.) (2011). Estrategia en Salud Mental del Sistema Nacional de Salud, 2009. Madrid: Ministerio de Sanidad y Consumo.

Gómez, R. (2002). Pisos tutelados y pensiones concertadas, ¿̇una respuesta adecuada? En IMSERSO. Jornadas sobre Salud Mental: Problemas de Adaptación e Integración Social de la persona con enfermedad mental crónica, (pp. 299320). Madrid: Autor.

González López, A.C. (coord.) (2011). Plan de Salud Mental del Principado de Asturias 2011-2016. Oviedo: Gobierno del Principado de Asturias.

Grupo de trabajo de la guía de práctica clínica de intervenciones psicosociales en el trastorno mental grave (2009). Guía de Práctica Clínica de Intervenciones Psicosociales en el Trastorno Mental Grave. Madrid: Plan de Calidad para el Sistema Nacional de Salud del Ministerio de Sanidad y Política Social. Instituto Aragonés de Ciencias de la Salud.

Hansson, L. et al. (2002). Living situation, subjective quality of life and social network among individuals with schizophrenia living in community settings. Acta Psychiatrica Scandinavica, 105, pp. 343-350.

Jiménez, J.F. et al. (2000). Evaluación del funcionamiento de la vida diaria en personas con trastorno mental de larga evolución. Adaptación y fiabilidad de la versión española del "Basic Everyday Living Skills" (BELS). Actas Españolas de Psiquiatría, 28 (5), pp. 284-288.

Jordá, E. y Espinosa, J. (1990). El proceso de desinstitucionalización en la provincia de Valencia: estudio de los alojamientos utilizados mediante la escala de prácticas restrictivas. Revista Psiquiátrica de la Facultad de Medicina de Barcelona, 17 (2), pp. 77-87.

Jordá, E. \& Espinosa, J. (1991). Study of alternative accommodation, using the hospital hostel practices profile. Soc Psychiatry Psychiatr Epidemiol, 26, pp. 151-156.

Kallert, T., Leisse, M. \& Winiecki, P. (2007). Comparing the effectiveness of different types of supported housing for patients with chronic schizophrenia. Journal of Public Health, 15 (7), pp. 29-42.

Kyle, T. \& Dunn, J.R. (2008). Effects of housing circumstances on health, quality of life and healthcare use for people with severe mental illness: a review. Health and Social Care in the Community, 16 (1), pp. 1-15.

Lascorz, D., Serrats, E., Pérez V., Fábregas, J. y Vegué, J. (2009). Estudio comparativo coste-eficiencia en un dispositivo residencial para enfermos con trastorno mental severo. Revista AEN, XXIX, 103, pp. 191-201.

Leff, J., Trieman, N. y Gooch, C. (1996). Team for the assessment of psychiatric services (TAPS) project 33: prospective follow-up study of long stay patients discharged from two psychiatric hospitals. American Journal of Psychitary, 153 (10), pp. 1318-1324.

López Álvarez, M. et al. (2004). Los programas residenciales para personas con trastorno mental severo. Revisión y propuestas. Archivos de Psiquiatría, 67 (2), pp. 101-128.

López Álvarez, M. et al. (2005a). Evaluación del Programa Residencial para personas con trastorno mental severo en Andalucía (I): descripción general del programa y del estudio. Rehabilitación Psicosocial, 2 (7), pp. 2-15.

López Álvarez, M. et al. (2005b). Evaluación del Programa Residencial para personas con trastorno mental severo en Andalucía (II): características de los dispositivos residenciales. Rehabilitación Psicosocial, 2 (7), pp. 16-27.

López Álvarez, M. et al. (2005c). Evaluación del Programa Residencial para personas con trastorno mental severo en Andalucía (IV): perfiles funcionales y redes sociales de los residentes. Rehabilitación Psicosocial, 2 (2), pp. 44-55.

López Álvarez, M. y Laviana Cuetos, M. (2007). Rehabilitación, apoyo social y atención comunitaria a personas con trastorno mental grave. Propuestas desde Andalucía. Revista AEN, XXVII, 99, pp. 187-223.

Macpherson, R., Shepherd, G. y Edwards, T. (2004). Supported accommodation for people with severe mental illness: a review. Advances in Psychiatric Treatment, 10, pp. 180-188.

Maestro Barón, J.C. et al. (2001). Estudio de las alternativas residenciales para pacientes desinstitucionalizados en Granada y Sevilla. Anales de Psiquiatría, 17 (4), pp. 143-152.

Mares, A.S., Young, A.S., Mcguire, J.F. y Rosenheck, R.A. (2002). Residential environment and quality of life among seriously mentally ill residents of board and care homes. Community Mental Health Journal, 38 (6), pp. 447-458. 
Mark, M., Henry, G. y Julnes, G. (2000). Evaluation: An integrated framework for understanding, guiding, and improving policies and programs. San Francisco: Jossey-Bass.

Newman, S. y Goldman, H. (2008). Putting Housing First, Making Housing Last: Housing Policy for Persons with Severe Mental Illness. American Journal of Psychiatry, 165 (10), pp. 1242-1248.

O 'Driscoll, C. y Leff, J. (1993).The TAPS Project 8: design of the research study on the long-stay patients. British Journal of Psychiatry, 162 (suppl. 19), pp. 18-24.

Ogilvie, R.J. (1997). The state of supported housing for mental health consumers: A literature review. Psychiatric Rehabilitation Journal, 21 (2), pp. 122-131.

Piat M. y Sabetti J. (2011). Residential Housing for Persons with Serious Mental Illness: The Fifty Year Experience with Foster Homes in Canada. En J.H. Stone y M. Blouin, (eds.). International Encyclopedia of Rehabilitation. Recuperado de http://cirrie.buffalo.edu/encyclopedia/en/article/236/.

Priebe, S. et al. (2005). Reinstitutionalisation in mental health care: comparison of data on service provision from six European countries. British Medical Journal, 330, pp. 123-126.

Rickard, C. et al. (2002). Residential care for mentally ill people in Andalusia and London ñ a comparison of care environments, usersí attitudes and cost of care. Journal of Mental Health, 11 (3), pp. 327-337.

Servicio de Salud del Principado de Asturias. (ed.) (1998). Plan de Atención Psiquiátrica y Salud Mental para Asturias. Oviedo: Servicio de Publicaciones del Principado de Asturias.

Shepherd, G. \& Murray, A. (2001). Residential care. En G. Thornicroft y G. Szmukler (eds). Textbook of Community Psychiatry (pp.309-20).Oxford: Oxford University Press.

Sörgaard, K.W. et al. (2001). Predictors of social relations in persons with schizophrenia living in the community: a Nordic multicentre study. Social Psychiatry and Psychiatric Epidemiology, 36, pp. 13-19.

Varela Crespo, L. (2010). La educación social y los servicios sociales en los procesos de desarrollo comunitario: revitalización del trabajo en red. Pedagogía Social. Revista Interuniversitaria, 17, pp. 137-148.

\section{Notas}

${ }^{1}$ Esta investigación ha sido subvencionada por el Gobierno del Principado de Asturias con cargo a fondos provenientes del Plan de Ciencia, Tecnología e Innovación (P.C.T.I.) de Asturias. Referencia BPo6-083.

\section{Agradecimientos}

Traducción elaborada por Bruno Costa

\section{Dirección de los autores}

Omar García Pérez. Universidad de Oviedo. Departamento de Ciencias de la Educación. C/ Aniceto Sela, s/n. 33005 Oviedo

Correo electrónico: garciaomar@uniovi.es

Fecha de recepción del artículo: 7.6.2012

Fecha de revisión del artículo: 20.2.2013

Fecha de aceptación final: 4.3.2013

\section{Cómo citar este artículo}

García-Pérez, О. (2013). Viviendas supervisadas para personas con trastorno mental severo en Asturias: ¿̇ambiente restrictivo o abiertas a la comunidad?. Pedagogía Social. Revista Interuniversitaria, 22, pp. 123-136 